\title{
Reconsolidation Allows Fear Memory to Be Updated to a Less Aversive Level through the Incorporation of Appetitive Information
}

\author{
Josue Haubrich ${ }^{1,2}$, Ana P Crestani' ${ }^{1,2}$, Lindsey F Cassini ${ }^{1,2}$, Fabiana Santana ${ }^{1,2}$, Rodrigo O Sierra ${ }^{1,2}$, \\ Lucas de $O$ Alvares ${ }^{1,2,3}$ and Jorge A Quillfeldt*, ${ }^{*, 2,3}$ \\ 'Psychobiology and Neurocomputation Lab, Department of Biophysics, Federal University of Rio Grande do Sul, Porto Alegre, Brazil; \\ ${ }^{2}$ Neurosciences Graduate Program, Federal University of Rio Grande do Sul, Porto Alegre, Brazil
}

\begin{abstract}
The capacity to adapt to new situations is one of the most important features of memory. When retrieved, memories may undergo a labile state that is sensitive to modification. This process, called reconsolidation, can lead to memory updating through the integration of new information into a previously consolidated memory background. Thus reconsolidation provides the opportunity to modify an undesired fear memory by updating its emotional valence to a less aversive level. Here we evaluated whether a fear memory can be reinterpreted by the concomitant presentation of an appetitive stimulus during its reactivation, hindering fear expression. We found that memory reactivation in the presence of appetitive stimuli resulted in the suppression of a fear response. In addition, fear expression was not amenable to reinstatement, spontaneous recovery, or rapid reacquisition. Such effect was prevented by either systemic injection of nimodipine or intra-hippocampal infusion of ifenprodil, indicating that memory updating was mediated by a reconsolidation mechanism relying on hippocampal neuronal plasticity. Taken together, this study shows that reconsolidation allows for a 're-signification' of unwanted fear memories through the incorporation of appetitive information. It brings a new promising cognitive approach to treat fear-related disorders.

Neuropsychopharmacology (20I5) 40, 315-326; doi:I0.1038/npp.20I4.174; published online I3 August 20I4
\end{abstract}

\section{INTRODUCTION}

Previously established memories might become labile following retrieval, requiring to be reconsolidated in order to endure (Nader et al, 2000). It has been suggested that the functional role of reconsolidation is to allow new information to be integrated into the background of an established memory (Besnard et al, 2012; Lee, 2009; Rodriguez-Ortiz et al, 2005), retaining its predictive and adaptive relevance. Supporting this view, reconsolidation-mediated updating was reported in human episodic memory paradigms (Forcato et al, 2010; Hupbach et al, 2007) and in animal studies using aversive (Monfils et al, 2009; De Oliveira Alvares et al, 2013; Rao-Ruiz et al, 2011; Sierra et al, 2013) and appetitive paradigms (Olshavsky et al, 2013b).

\footnotetext{
*Correspondence: Dr JA Quillfeldt, Laboratório de Psicobiologia e Neurocomputação (Psychobiology and Neurocomputation Lab), Departamento de Biofísica, Instituto de Biociências, Universidade Federal do Rio Grande do Sul (UFRGS), Avenue Bento Gonçalves 9500, Prédio 43422, Sala 208, CEP, Porto Alegre, Rio Grande do Sul 9|50।-970, Brazil, Tel: +55 5 I 3308 7604, Fax: +55 5 I 3308 7003, E-mail: quillfe@ufrgs.br

${ }^{3}$ These authors contributed equally to this work.

Received 27 October 2013; revised II June 2014; accepted 26 June 20।4; accepted article preview online 15 July 2014
}

Therapeutic approaches to fear-related disorders such as posttraumatic stress disorder (PTSD) aim to hinder aversive responses expressed in the presence of trauma-related cues (Cukor et al, 2009). Reconsolidation provides a promising strategy to modify undesired fear memories in such psychiatric conditions, by updating its emotional valence to a less aversive level. As reconsolidation allows permanent changes in the memory trace (Díaz-Mataix et al, 2011; Doyère et al, 2007; Monfils et al, 2009; Nader et al, 2000), it could be a more effective strategy than extinction-based therapies-in which fear responses often return by either the passage of time (spontaneous recovery) or exposure to the unconditioned stimulus (reinstatement). It happens because extinction does not disrupt the original memory trace but induces new learning that transiently inhibits fear expression (Archbold et al, 2010; Bouton et al, 2012; Rescorla and Heth, 1975).

Recent studies have explored the reconsolidation process as a therapeutic target, focusing on memory-related fear and anxiety (Blundell et al, 2008; Gamache et al, 2012; Parsons and Ressler, 2013; Steckler and Risbrough, 2012; Stern et al, 2012). However, their approaches rely mostly on pharmacological interventions aiming to disrupt reconsolidation. Nonetheless, it may be problematic for human application, as most of these pharmacological agents would require intracranial administration, and some of them are 
toxic. On the other hand, the possibility to modify memory through reconsolidation updating mechanisms without any drug provides a much safer therapeutic approach, as previously reported (Monfils et al, 2009; Rao-Ruiz et al, 2011; Schiller et al, 2010). As reconsolidation allows changes in the memory content through the incorporation of new information (De Oliveira Alvares et al, 2013), it may be able to update fear memories to a less aversive state.

The association between neutral (eg, context) and emotional stimuli (eg, footshock or food) elicits memories of both positive and negative valence. After learning, the brain produces negative affective reactions, such as fear, when re-exposed to neutral stimuli that predict pain; and positive reactions, such as pleasure, when re-exposed to stimuli that predict rewards (Gross and Canteras, 2012; Robinson and Berridge, 2013). A recent study has shown that an aversive learned cue for unpleasantness can become desirable when rats were placed in a different physiological state (Robinson and Berridge, 2013), suggesting that emotional valence can be an important target for memory modifications.

Here we hypothesize that if a contextual fear memory is reactivated in the presence of an appetitive stimuli, such positive information could be incorporated through reconsolidation, retuning its emotional valence to a less aversive level. The updated memory would then contain two emotionally opposite associations-a negative context/shock and a positive context/appetitive association. Our findings show that animals exposed to appetitive stimuli during memory reactivation exhibited a robust and long-lasting decrease in fear expression, confirming our hypothesis.

\section{MATERIALS AND METHODS}

\section{Subjects}

Male (weighing 210-260 g) and female (180-230 g) Wistar rats from our breeding colony, aged 60-70 days, were used. Animals were housed in plastic cages, 4-5 per cage, with water and food available ad libitum. Female subjects were not classified according to their estrous cycle phase, just randomly pooled in the groups. All experiments were performed in accordance with the national animal care legislation and guidelines (Brazilian Law 11794/2008) and approved by the University's Ethics Committee.

\section{Appetitive Stimuli}

In the first experiment, different types of palatable food were used as appetitive stimuli: chocolate (milk chocolate, Garoto), sugar cubes, or sweetened cereal (Froot-Loops, Kellogg's). In the remaining experiments, only chocolate was used as appetitive stimulus.

\section{Habituation to Appetitive Stimuli}

For 3 consecutive days, animals were habituated in their homecages to one of the available appetitive stimuli consisting of 4-h exposure/day with $5 \mathrm{~g}$ (in chunks) of the respective palatable food available per animal. Food was fully consumed in this period. In the first experiment, an additional control group was habituated to neutral objects the same size and shape of the chocolate chunks $(4 \times 3 \times$ $1 \mathrm{~cm}^{3}$ aluminum blocks).

\section{Contextual Fear Conditioning (CFC)}

Forty-eight hours after the last habituation, rats were trained on CFC. The CFC chamber consisted of an illuminated Plexiglas box $\left(25.0 \times 25.0-\mathrm{cm}^{2}\right.$ grid of parallel $0.1-\mathrm{cm}$ caliber stainless steel bars spaced $1.0 \mathrm{~cm}$ apart). In the conditioning (training) session, rats were placed in the chamber for $3 \mathrm{~min}$ and then received two 2 -s, $0.5-\mathrm{mA}$ footshocks separated by a 30-s interval. Animals were kept in the conditioning environment for an additional minute before returning to their homecages.

\section{Memory Reactivation and Test}

Memory reactivation sessions consisted of 3-min re-exposure to the conditioned context. Reactivations were conducted three times, at days 3, 5, and 7 after training. In the experiment 2 , two additional groups were submitted to a single 3- or 9-min reactivation session 5 days after training. In the experiment 6 , a group of animals was reactivated on days 30, 32, and 34 after training. During the reactivation sessions of the appetitive stimuli groups, palatable food (chocolate, sugar, or sweetened cereal) were placed on the grid floor of the CFC chamber, and animals were able to explore and eat them. Controls were reactivated in the context without any appetitive stimuli. In the first experiment, an additional control group was exposed to a neutral stimulus instead of the appetitive stimulus. In the experiment 3 , an additional control was exposed to the appetitive stimulus in a standard empty homecage (3-min exposures on days 3, 5, and 7 after training-unpaired appetitive stimulus group). In experiment 4, reactivation sessions of both the control and the appetitive stimulus groups were conducted in a novel context that did not share any attribute with the training one, as previously described (De Oliveira Alvares et al, 2012). In the experiment 5, the appetitive stimulus was presented immediately after the reactivation sessions, during 3-min in another chamber (standard homecage without roommates).

During the test (10 days after training in all experiments, except on experiment 6 , in which a group was tested 37 days after training), animals were re-exposed to the training context for 4 min without any food. In the experiment 1 , animals received two immediate 2 -s $0.5-\mathrm{mA}$ non-paired footschocks (in a $15 \times 10 \times 11.5 \mathrm{~cm}^{3}$ blue polypropylene box) $24 \mathrm{~h}$ after test and were retested for reinstatement in the following day. In the experiment 2, animals were retested 21 days after the first test in order to check for spontaneous recovery. In the experiment 3, after a 4-min test session, animals received a $2-\mathrm{s}, 0.25-\mathrm{mA}$ footshock and were removed from the context after additional $30 \mathrm{~s}$ (weak retraining sessions). Twenty-four hours later, they were retested for savings effect. Freezing behavior was always measured by a blind researcher.

\section{Stereotaxic Surgery and Cannulae Implantation}

Animals used in the experiment 6 were anesthetized by ketamine and xylazine (75 and $10 \mathrm{mg} / \mathrm{kg}$, respectively). 
Guide cannulae were bilaterally implanted at AP $-4.2 \mathrm{~mm}$ (from bregma), LL $\pm 3.0 \mathrm{~mm}, \mathrm{DV} 1.8 \mathrm{~mm}, 1.0 \mathrm{~mm}$ above the CA1 area of the dorsal hippocampus (Supplementary Figure S4). Behavioral procedures were performed 1 week after surgery. Animals with inaccurate cannulae position were excluded from the statistical analysis.

\section{Drugs}

Glucose (Sigma) was dissolved in sterile isotonic saline to a concentration of $100 \mathrm{mg} / \mathrm{ml}$. Glucose or its vehicle were injected intra-peritoneally immediately before the reactivation sessions. The total volume injected was $1 \mathrm{ml} / \mathrm{kg}$.

Protein synthesis inhibitor cycloheximide (Sigma) was dissolved in $1 \%$ sterile isotonic saline with $1 \%$ dimethylsulfoxide to a concentration of $2.2 \mathrm{mg} / \mathrm{ml}$. Cycloheximide or its vehicle were injected intra-peritoneally immediately after the reactivation. The total volume injected was $1 \mathrm{ml} / \mathrm{kg}$.

The L-type voltage-gated calcium channels (LVGCCs) antagonist nimodipine (Sigma) was dissolved in sterile isotonic saline with $8 \%$ dimethylsulfoxide to a concentration of $16 \mathrm{mg} / \mathrm{ml}$. Nimodipine or its vehicle was injected subcutaneously $30 \mathrm{~min}$ before the reactivation sessions. The total volume injected was $1 \mathrm{ml} / \mathrm{kg}$.

Ifenprodil (Sigma), the selective antagonist of the GluN2B $\mathrm{NMDA}_{\mathrm{R}}$ subunit, was dissolved in a phosphate-buffered saline to a concentration of $1 \mathrm{mg} / \mathrm{ml}$. Ifenprodil or its vehicle was infused intrahippocampally at a slow rate $(20 \mu \mathrm{l} / \mathrm{h} ; 0.5 \mu \mathrm{l} /$ side), $15 \mathrm{~min}$ before the reactivation sessions.

\section{Glycemia}

Blood was collected from the tip of the tail, and glycemia was assessed by On-Call Plus Blood Glucose Monitoring System glucometer (Acon Labs Inc., San Diego, USA).

\section{Data Analysis}

Memory was measured quantifying freezing behavior and expressed as percentage of the total session time. Reactivation data was analyzed using Student's $t$-test or repeatedmeasures' ANOVA followed by Tukey's post-hoc test. Fear conditioning tests and glucose blood levels measurements were analyzed using Student's $t$-test, one-way ANOVA or two-way ANOVA followed by Tukey's post-hoc test.

\section{RESULTS}

\section{Experiment 1: Repeated Reactivations in the Presence of Appetitive Stimuli Lead to the Attenuation of Fear Memory}

It has been suggested that reconsolidation enables memory updating by adding new information into the background of a previously stored memory (Lee, 2009). Here we asked whether fear memory updating could modify its emotional valence to a less aversive level.

In order to induce reconsolidation, rats were fear conditioned (CFC), and later, memory was reactivated three times by 3-min context re-exposures. In the appetitive stimuli groups, palatable food (chocolate, sugar, or sweetened cereal) were present inside the conditioned context during reactivations. Control groups were carried out in the absence of food or in the presence of a neutral stimulus during reactivations (Figure 1a). We hypothesized that reactivation in the presence of an appetitive stimulus would favor fear memory reinterpretation, changing its valence into a less aversive level.

During reactivations sessions (Figure $1 \mathrm{~b}$ ), repeatedmeasures ANOVA revealed no effect of session $\left(\mathrm{F}_{(2,50)}=\right.$ $0.257, P=0.774)$ or session $\times$ group interaction $\left(\mathrm{F}_{(8,50)}=\right.$ $0.425, \quad P=0.901)$ but significant effect of group $\left(\mathrm{F}_{(4,25)}=10.66, P<0.001\right)$. Tukey's post-hoc indicated that rats from the appetitive stimulus group showed lower freezing levels compared with both animals reactivated without any food (chocolate: $P=0.001$; cereal group: $P<0.001$; sugar group: $P=0.021$ ) and animals reactivated in the presence of neutral objects (chocolate group: $P=0.003$; cereal group: $P=0.002$ ), with the exception of the sugar group $(P=0.09)$. Hence, the presence of appetitive stimuli during memory reactivation was able to hinder fear expression.

In the test session, one-way ANOVA revealed significant differences among the groups ((Figure 1c; $\mathrm{F}_{(4,25)}=5.66$, $P<0.002)$. Tukey's post-hoc indicated that subjects reactivated in the context containing chocolate or cereal, but not sugar, persisted expressing lower freezing levels in comparison with those reactivated without any food (chocolate group: $P=0.036$; cereal group: $P=0.014$; sugar group: $P=0.31)$ and those reactivated with neutral objects (chocolate group: $P=0.029$; cereal group: $P=0.011$; sugar group: $P=0.27)$. These data suggest that the repeated reactivation in the presence of some appetitive stimulus (chocolate and cereal, but not sugar) enabled memory to incorporate the emotionally positive information, updating its emotional content to a less aversive level.

Extinction is a form of new learning that is effective in inhibiting conditioned fear responses. However, it fails to suppress fear memory permanently due to reinstatement, spontaneous recovery, and rapid reaquisition (Bouton et al, 2006). In contrast, memory updating would possibly allow permanent changes on fear expression. Thus, we evaluated whether an unpaired US (footshock) presentation would reinstate fear expression. In the retest after the reinstatement procedure (Figure 1d), one-way ANOVA showed significant differences among groups $(\mathrm{F}(4,25)=$ 6.52, $P<0.001)$. Tukey's post-hoc test revealed that animals reactivated in the presence of chocolate or cereals persisted in exhibiting lower freezing levels in comparison with those reactivated without any food (chocolate group: $P=0.01$; cereal group: $P=0.017$; sugar group: $P=0.407)$ and in the presence of neutral stimulus (chocolate group: $P<0.01$; cereal group: $P=0.011$; sugar group: $P=0.33$ ), similarly as was observed in the first test.

Accordingly, fear expression attenuation induced by appetitive stimuli seems to be permanent. These results suggest that memory emotional content was effectively modified (updated) rather than transiently inhibited.

\section{Experiment 2-Appetitive Stimulus-Induced Memory Updating is Long-Lasting and not Gender-Specific}

In the first set of experiments, CFC memory reactivation in the presence of appetitive stimuli was able to suppress fear 

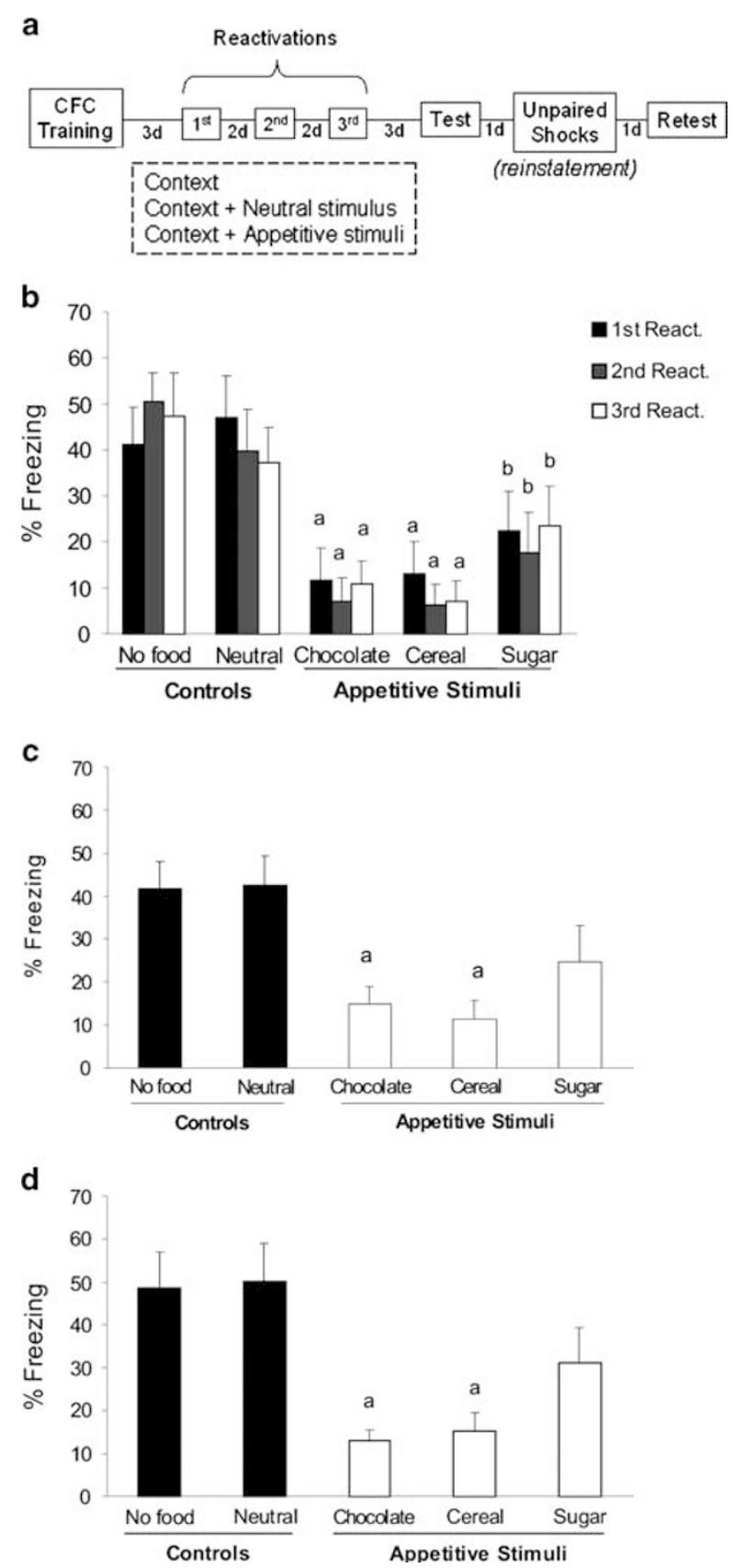

Figure I Repeated reactivations in the presence of appetitive stimuli lead to the attenuation of a fear memory. (a) Schematic representation of the experimental procedures. Fear-conditioned rats were submitted to 3-min memory reactivation sessions on days 3, 5, and 7 after training. Reactivations took place in the conditioned context without any food (controls, $n=6)$ or in the presence of neutral stimulus $(n=6)$ or an appetitive stimulus (chocolate, sweetened cereal, or sugar, $n=6$ per group). On day 10, all groups were tested. On day II, all groups received two 2-s non-paired footshock (reinstatement) followed by a retest $24 \mathrm{~h}$ later. (b) Freezing levels during reactivation sessions. (c) Freezing levels during test. (d) Freezing levels during the retest conducted $24 \mathrm{~h}$ after the reinstatement procedure. Data expressed as mean + SEM of the percentage of freezing time during reactivations or test. ${ }^{a}$ Significantly different from the no food and neutral groups $(P<0.05$, ANOVA followed by Tukey's post-hoc test). 'Significantly different from the no food group $(P<0.05$, Tukey's post-hoc test). with no reinstatement. These results indicate that fear inhibition was achieved through the update of fear memory with hedonic information, leading to a modified, less aversive memory. We next evaluated whether the fear suppression induced by our protocol would be long-lasting or prone to spontaneous recovery. Accordingly, animals were submitted to the same procedures described above and were retested 21 days after the first test (Figure 2a).

During reactivations (Figure $2 \mathrm{~b}$, left side), repeatedmeasures ANOVA revealed no effect of session $\left(\mathrm{F}_{(2,34)}=\right.$ $0.787, P=0.463)$ or session $\times$ group interaction $\left(F_{(2,34)}=\right.$ $0.798, \quad P=0.458)$ but significant effect of group $\left(\mathrm{F}_{(1,17)}=9.67, P<0.01\right)$, indicating that animals exposed to the appetitive stimulus displayed lower freezing levels than controls. In the first test (Figure 2c, left side) and in the remote test (Figure 2d, left side), animals from the appetitive stimulus group kept expressing lower freezing levels (Student's $t$-test; $t_{(17)}=5.29, P=0.034$ and $t_{(11)}=2.89$, $P=0.015$, respectively). Therefore animals reactivated in the presence of appetitive stimulus showed long-lasting fear suppression, with no spontaneous recovery.

As the previous experiments were conducted with females, we replicated the last experiment in males from the same breeding colony in order to exclude the possibility of a gender-specific phenomenon. Not only freezing levels were very similar between genders, but also male rats displayed the same general response pattern. During reactivations (Figure $2 \mathrm{~b}$, right side), repeated-measures ANOVA revealed no effect of session $\times$ group interaction $\left(\mathrm{F}_{(1,13)}=0.206, P=0.658\right)$ but significant effect of session $\left(\mathrm{F}_{(1,13)}=13.559, \quad P=0.003\right)$ and group $\left(\mathrm{F}_{(1,17)}=9.67\right.$, $P<0.01)$, indicating that animals exposed to the appetitive stimulus displayed lower freezing levels than controls. In the first test (Figure $2 c$, right side) and in the remote test (Figure 2d, right side), animals from the appetitive stimulus group kept expressing lower freezing levels (Student's $t$-test; $t_{(13)}=3.61, P=0.003$ and $t_{(13)}=3.75, P=0.009$, respectively). Therefore animals reactivated in the presence of appetitive stimulus showed long-asting fear suppression, with no spontaneous recovery. Thus the emotional updating induced by this protocol seems to be independent from gender aspects.

The results of this section indicate that the emotional valence of the original fear memory can be altered by the concomitant presentation of appetitive information during multiple memory reactivation sessions, leading to a longlasting reduction on fear expression.

One could argue if only one reactivation would suffice to induce memory updating. Hence, we conducted an experiment where memory was reactivated once for 3 or $9 \mathrm{~min}$ (Supplementary Figure S1A in Supplementary Material). During the 3 -min reactivation, rats from the appetitive stimulus group showed significantly less freezing than controls (Student's $t$-test, $t_{(18)}=2.23, P=0.038$; Supplementary Figure S1B), but no significant difference was found between the groups in the test (Student's $t$ test, $t_{(18)}=0.39, P=0.7$; Supplementary Figure S1C). We hypothesized that a single 3-min reactivation session induced memory updating of little magnitude, leading to a subthreshold behavioral performance on test. Thus, we next tested for a 9-min reactivation session to allow for a more robust integration of information concerning the appetitive 
a

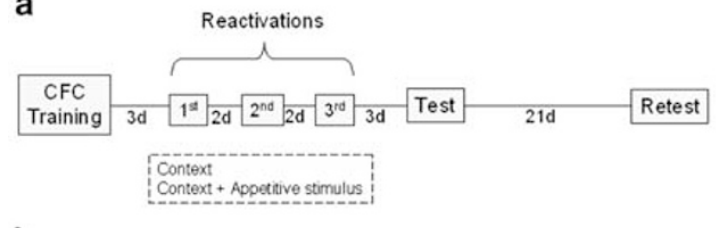

b 70
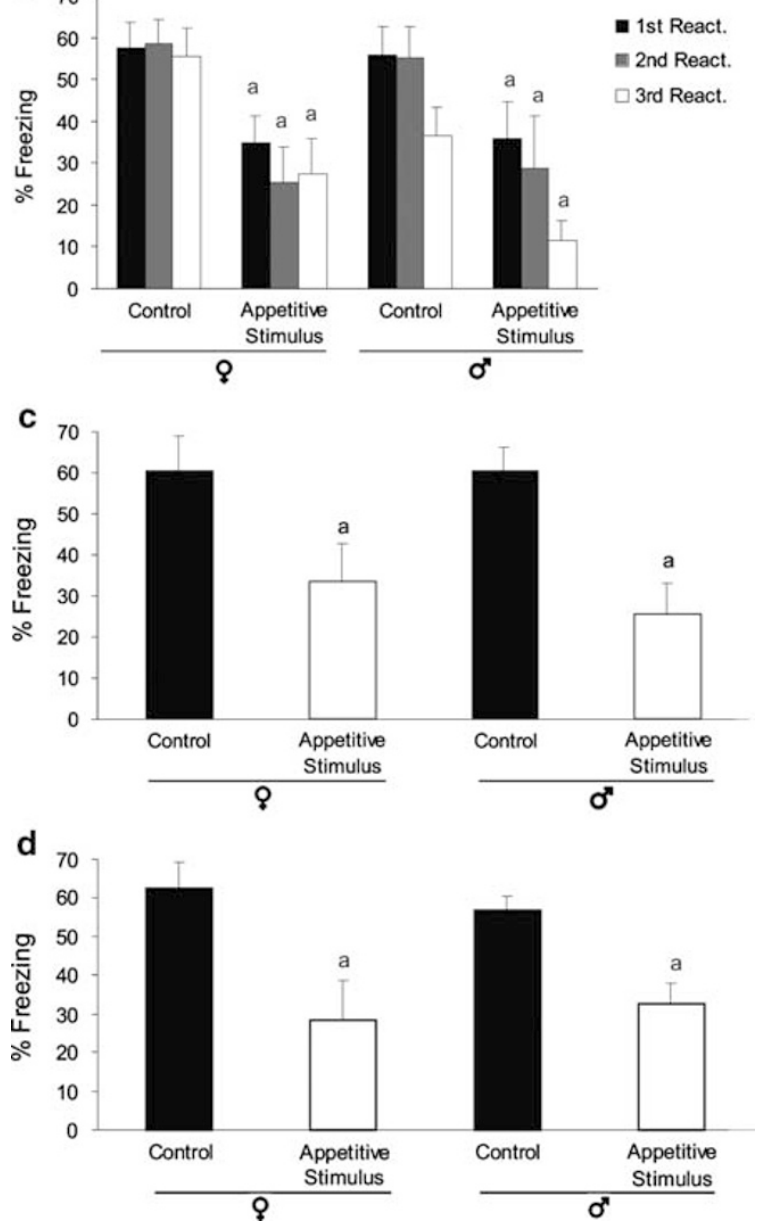

Figure 2 Memory updating induced by appetitive stimulus is long lasting and is not gender specific. (a) Schematic representation of the experimental procedures. Fear-conditioned male and female rats were submitted to memory reactivation sessions on days 3, 5, and 7 after training. Reactivations took place in the conditioned context without any food (control group; females: $n=9$; males: $n=8$ ) or in the presence of appetitive stimulus (females: $n=10$; males: $n=7$ ). On day 10, rats were tested and retested 21 days later. (b) Freezing levels during reactivation sessions. (c) Freezing levels during test. (d) Freezing levels during the retest. Data expressed as mean + SEM of the percentage of freezing time during reactivations or test sessions. ${ }^{a}$ Significantly different from the respective control group ( $P<0.05$, ANOVA followed by Tukey's post-hoc test).

stimulus (the 9-min length was not arbitrary, as it is the sum of the multiple short reactivations, and yet, it is not a continuous context re-exposure that could induce an extinction effect). During reactivation, the appetitive stimulus group showed significantly less freezing than controls (repeated-measures ANOVA; $\mathrm{F}_{(1,12)}=8.915$, $P=0.014$; Supplementary Figure S1D). No difference was detected among the three 3-min blocks within each group $\left(F_{(1,12)}=0.15, P=0.906\right)$. In the test session, rats reactivated in the presence of the appetitive stimulus remained showing less freezing than controls (Student's $t$-test, $t_{(11)}=3.4, P=0.006$; Supplementary Figure $\mathrm{S} 1 \mathrm{E}$ ). A retest was conducted 21 days later, and rats from the appetitive stimulus group did not show spontaneous recovery compared with controls (Student's $t$-test, $t_{(11)}=2.54$, $P=0.027$; Supplementary Figure S1F). Next, we tested the 9-min reactivation protocol for reinstatement (Supplementary Figure S1G). Again, the appetitive stimulus group showed significantly less freezing than controls during reactivation (repeated-measures ANOVA; $\mathrm{F}_{(1,13)}=22.304$, $P<0.001$; Supplementary Figure $\mathrm{S} 1 \mathrm{H}$ ) with no within-group difference $\left(\mathrm{F}_{(2,26)}=1.905, P=0.169\right)$. In the test, animals from the appetitive stimulus group maintained the low freezing levels (Student's $t$-test, $t_{(13)}=4.4, \quad P=0.001$; Supplementary Figure S1I), and fear was not reinstated in the retest (Student's $t$-test, $t_{(13)}=5.8, P>0.001$; Supplementary Figure S1J). Therefore these results show that memory updating can be also achieved with a single, yet longer, reactivation session in the presence of appetitive stimulus.

\section{Experiment 3-The Updated Memory does not Reacquire the Aversive Association after a Weak Reconditioning}

Next, we evaluated whether the fear expression of an updated memory would be reacquired after a weak retraining. Accordingly, animals were fear conditioned and underwent memory reactivations paired or not with an appetitive stimulus. An additional control group was not submitted to reactivation but exposed to the appetitive stimulus for the same amount of time and at the same timepoints (hour of the day) of the reactivations in the empty homecage (unpaired appetitive stimulus group). Also, a parallel group was submitted to a 30 -min extinction training instead of the multiple reactivations. In the test, freezing was assessed for $4 \mathrm{~min}$, and then animals received a weak footshock $(2 \mathrm{~s}, 0.25 \mathrm{~mA})$. In the next day, animals were retested for savings effect (Figure $3 \mathrm{a}$ ).

During reactivations, the appetitive stimulus group showed lower freezing levels than controls (repeatedmeasures ANOVA; $F_{(1,5)}=85.306, P<0.001$; Figure $3 b$ ). In the extinction training, repeated-measures ANOVA showed a significant time-dependent decrease on freezing $\left(\mathrm{F}_{(1,15)}=33.73, P=0.002\right.$; Figure $\left.3 \mathrm{c}\right)$. In the test session (Figure 3d), one-way ANOVA indicated significant differences among the groups $\left(\mathrm{F}_{(3,30)}=26.11, P<0.001\right)$. Tukey's post-hoc test revealed no difference between the control and unpaired appetitive stimulus group $(P=0.38)$. However, the appetitive stimulus and extinction groups expressed lower freezing levels than the other groups $(P<0.01)$.

In the savings test (Figure $3 \mathrm{e}$ ), one-way ANOVA indicated significant differences among the groups $\left(\mathrm{F}_{(3,30)}=10.05\right.$, $P<0.001)$. Tukey's post-hoc test revealed that the extinction group rapidly reacquired the contextual fear memory, expressing the same freezing levels than the control and unpaired appetitive stimulus groups $(P>0.05)$. On the other hand, the appetitive stimulus group persisted expressing lower freezing levels than the control groups $(P<0.05)$, despite not differing from the extinction group $(P=0.068)$. Importantly, repeated-measures ANOVA followed by 
a
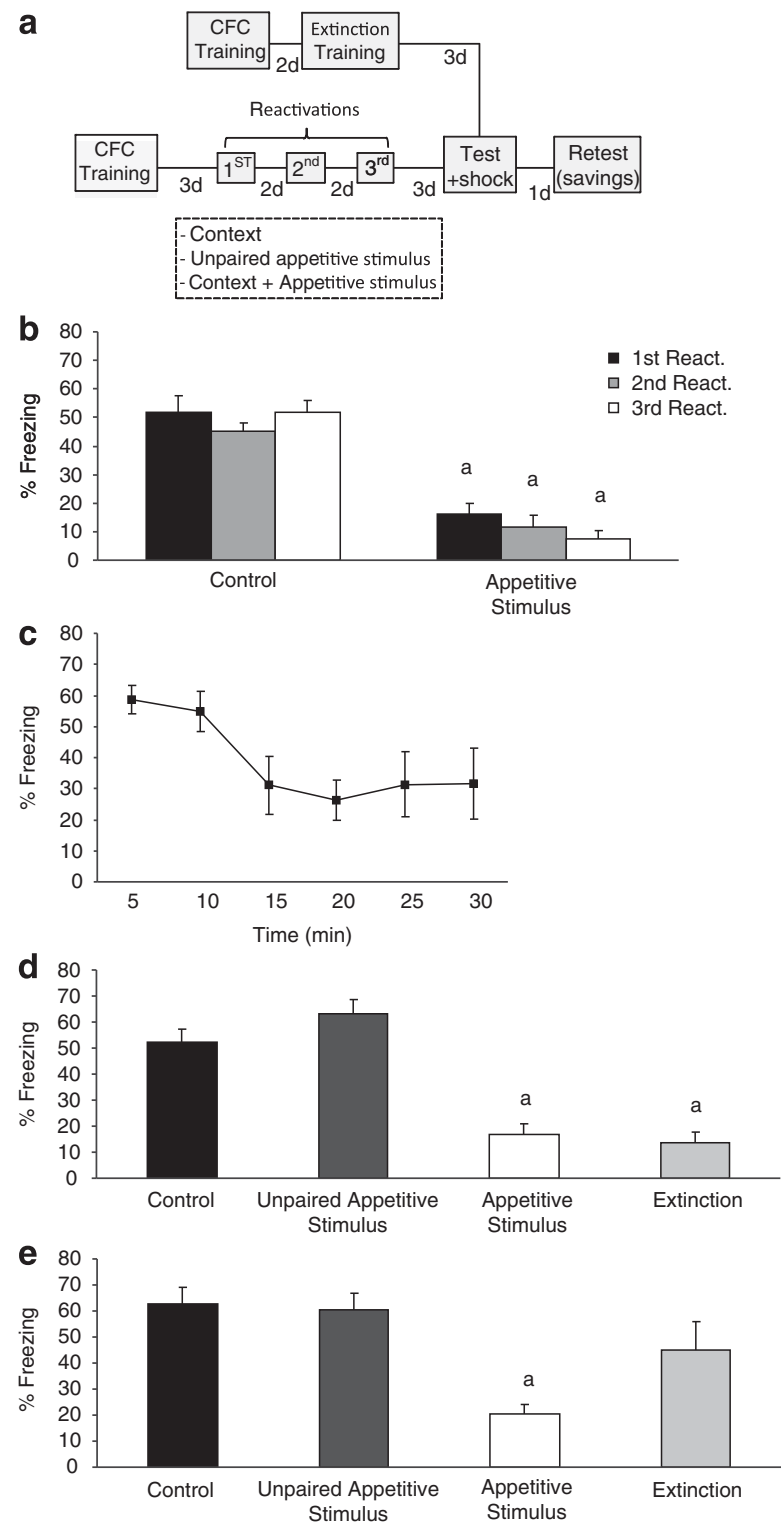

Figure 3 The updated memory does not reacquire the aversive association after a weak reconditioning. (a) Schematic representation of the experimental procedures. Fear-conditioned rats were submitted to memory reactivation sessions on days 3, 5, and 7 after training. Reactivations took place in the conditioned context without any food (control group; $n=8$ ) or in the presence of appetitive stimulus $(n=9)$. Another group was submitted to an extinction session on day $7(n=6)$. An additional control received the appetitive stimulus in an empty homecage (unpaired appetitive stimulus; $n=8$ ). On day 10 , rats were tested and then received a 2-s 0.25-mA footshock. On day II, animals were retested for savings. (b) Freezing levels during reactivation sessions. (c) Freezing levels during extinction training. (d) Freezing levels during test. (e) Freezing levels during the retest. Data expressed as mean + SEM of the percentage of freezing time during reactivations or test sessions. ${ }^{a}$ Significantly different from the respective control group $(P<0.05$, repeated-measures ANOVA followed by Tukey's post-hoc test). In the extinction training, there was a significant within-session difference (repeated-measures ANOVA, $P=0.02)$.

Tukey's post-hoc test found no difference between test and savings test session in the appetitive stimulus group $(P>0.05)$ but revealed a significant increase in fear expression in the extinction group $(P<0.001)$. This experi- ment shows that the emotional-updating procedure does not lead to savings, suggesting that memory valence was effectively re-evaluated.

\section{Experiment 4: Memory Reactivation is a Necessary Condition to the Update of its Emotional Content}

To verify whether the above effects are dependent on memory reactivation, fear-conditioned rats were exposed three times to a novel context containing appetitive stimulus (Figure $4 \mathrm{a}$ ). We predicted that this protocol would not be effective as fear memory would not be associated with the hedonic information.

As expected, there was no significant effect of session, group, and session $\times$ group interaction during reactivations (Figure 4b; Repeated-measures ANOVA $P>0.05$ ) and in the test (Figure 4c; Student's $t$-test, $t_{(11)}=0.05, P=0.96$ ). This result shows that memory requires to be reactivated in order to reconsolidate and has its emotional content updated with hedonic information.

\section{Experiment 5: Memory Valence Updating does not Depend on Nutritional Properties of the Appetitive Stimulus or on Glucose Levels}

Glycemia is known to have a significant role in learning and memory (Gold and Korol, 2012; Messier, 2004). All the studied appetitive stimuli are rich in glucose, and its ingestion may have induced changes in blood glucose levels. Accordingly, besides its hedonic property, appetitive stimuli consumption may have influenced memory through their nutritional effects.

To address these possibilities, we first trained an additional group of animals as described previously, but immediately after reactivation they were isolated in a standard homecage with access to chocolate for $3 \mathrm{~min}$. In this protocol, animals would ingest the edible appetitive stimulus, but the hedonic eating experience would not be contingent with the context.

During reactivations (Figure $5 \mathrm{~b}$, left side), repeatedmeasures ANOVA revealed significant effect of session $\left(\mathrm{F}_{(2,32)}=5.727, P=0.007\right)$ but no effect of session $\times$ group interaction $\left(\mathrm{F}_{(2,32)}=0.252, P=0.779\right)$ and group $\mathrm{F}_{(1,16)}=$ $0.122 P=0.732)$. In the test, no difference was found between the groups (Figure $5 \mathrm{c}$, left side; Student's $t$-test, $\left.t_{(16)}=0.589, P=0.564\right)$. This result shows that the appetitive stimulus must be presented concomitant with memory reactivation in order to be associated with the conditioned context.

Next, we investigated the effect of glucose injection before reactivations. We injected $100 \mathrm{mg} / \mathrm{kg}$ of glucose i.p, a dose previously shown to affect fear memory consolidation (Gold, 1986). During reactivations (Figure 5b, right side), repeated-measures ANOVA revealed no effect of session $\left(\mathrm{F}_{(2,30)}=3.304, P=0.050\right)$, group $\left(\mathrm{F}_{(1,16)}=0.122 P=0.732\right)$, and session $\times$ group interaction $\left(\mathrm{F}_{(2,30)}=0.214, P=0.808\right)$. In the test, no difference was found between the groups (Figure $5 c$, right side; Student's $t$-test, $t_{(15)}=-0.883$, $P=0.391)$. These data indicate that the results described in experiments 1 and 2 were attributed to the hedonic impact of the appetitive stimuli rather than the glucose contained in the palatable foods. 
a

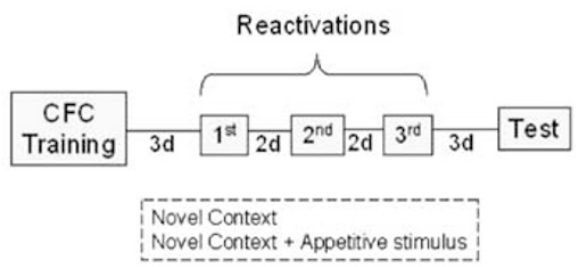

b
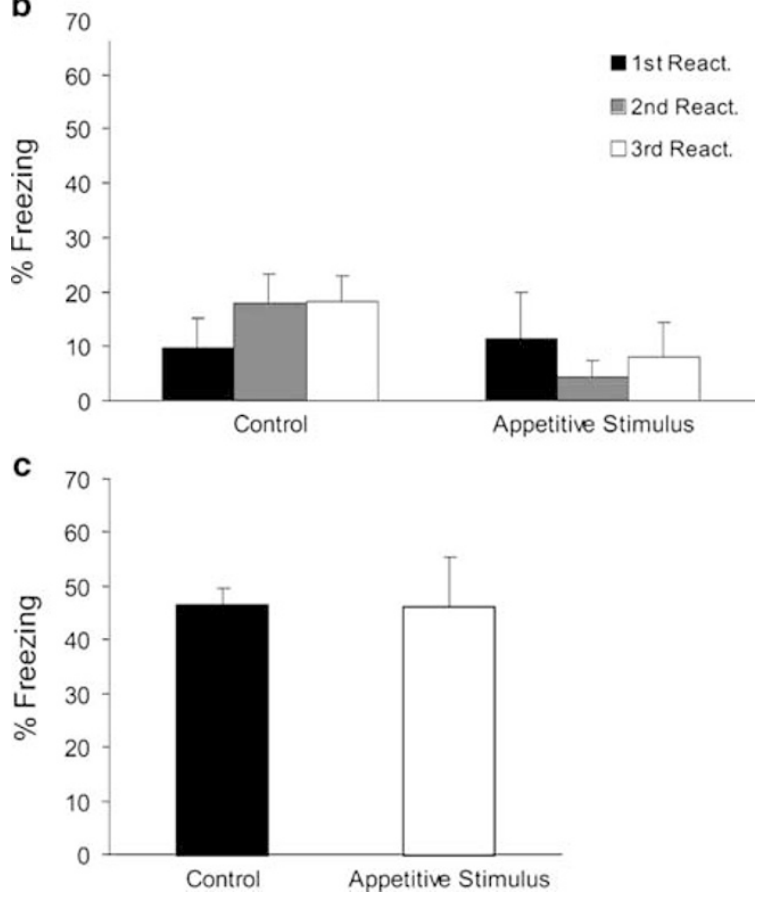

Figure 4 Memory reactivation is a necessary condition to the update of its emotional content. (a) Schematic representation of the experimental procedures. Fear-conditioned rats were submitted to memory reactivation sessions on days 3, 5, and 7 after training. Reactivations took place in a novel context containing appetitive stimulus $(n=6)$ or not (control group, $n=7$ ). On day 10, all groups were tested in the conditioned context. (b) Freezing levels during reactivation sessions. (c) Freezing levels during test. Data expressed as mean + SEM of the percentage of freezing time during reactivations or test sessions. There were no significant differences between groups or between sessions in each group.

We also investigated whether chocolate intake would significantly alter the glycemia $(1.4 \mathrm{~g} \pm 0.47$ at the third reactivation; Supplementary Table S1A in Supplementary Material). Accordingly, glycemia were measured before and $30 \mathrm{~min}$ after appetitive stimulus consumption $(2 \mathrm{~g}$ of chocolate) or $100 \mathrm{mg} / \mathrm{kg}$ i.p. glucose injection. Neither the consumption of the appetitive stimulus nor glucose injections were able to significantly affect the glycemic levels (see Supplementary Table S1B). Taken together, these results suggest that memory updating is caused by the hedonic proprieties of the appetitive stimuli rather than its nutritional/energetic properties.

\section{Experiment 6: Memory Valence Updating Involves Reconsolidation as it is Mediated by LVGCCs and Hippocampal GluN2B-Containing NMDA Receptors}

An established memory reactivation might undergo a labile state, requiring reconsolidation in order to persist or be a
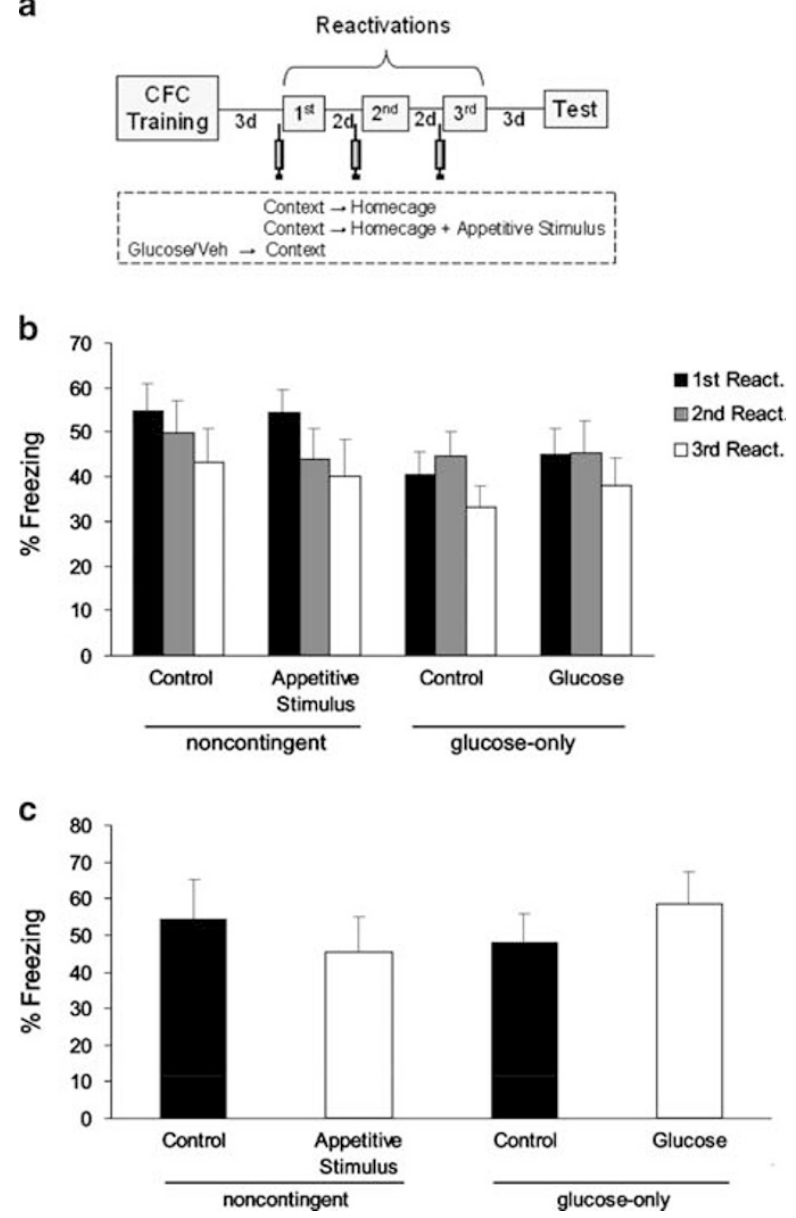

Figure 5 Memory valence updating does not depend on nutritional properties of the appetitive stimulus or on glucose levels. (a) Schematic representation of the experimental procedures. Fear-conditioned rats were submitted to memory reactivation sessions on days 3, 5, and 7 after training. Immediately after reactivations, a group of animals (non-contigent) were left in an empty homecage containing the appetitive stimulus $(n=9)$ or not $(n=9)$. Another group of animals (glucose-only) were submitted to the same procedure, but instead of being left in the empty homecage, they received i.p. injections of saline $(n=8)$ or $100 \mathrm{mg} / \mathrm{kg}$ glucose $(n=9)$ immediately before reactivations. On day 10, all groups were tested for 4 min. (b) Freezing levels during reactivation sessions. (c) Freezing levels during test. Data expressed as mean + SEM of the percentage of freezing time during reactivations or test sessions. There were no significant differences between groups.

updated (Nader et al, 2000). Previous studies have reported that the LVGCC activation is required for destabilization of reactivated memory (Suzuki et al, 2008; De Oliveira Alvares et al, 2013) and requires de novo protein synthesis to be reconsolidated (Nader et al, 2000). Hence, first we conducted an experiment to know whether our 3-min reactivation protocol was effective in inducing the memory destabilization-reconsolidation process.

Rats were fear conditioned and submitted to a reactivation session 3 days later. In order to disrupt reconsolidation, protein synthesis inhibitor cycloheximide was injected immediately after reactivation. The LVGCC antagonist nimodipine was also injected $30 \mathrm{~min}$ before reactivation in order to block memory destabilization/reconsolidation. Animals were tested for $4 \mathrm{~min} 48 \mathrm{~h}$ later (Supplementary 
Figure S2A). During the reactivation session (Supplementary Figure S2B), all the groups exhibited similar freezing levels (two-way ANOVA; $\mathrm{F}_{(1,46)}=7.42, P>0.45$ ). In the test session (Supplementary Figure S2C), two-way ANOVA revealed a significant effect of cycloheximide $\left(\mathrm{F}_{(1,46)}=7.42, P=0.009\right)$ and a cycloheximide $\times$ nimodipine interaction $\left(\mathrm{F}_{(1,46)}=5.25, P=0.027\right)$. Tukey's post-hoc test indicated that cycloheximide/vehicle group expressed lower freezing levels than the other groups $(P<0.05)$. These data showed that cycloheximide was able to disrupt memory, an effect that was prevented by nimodipine. This confirms that, following a 3-min reactivation session, memory undergoes a destabilization-reconsolidation process.

In addition, we evaluated the effect of cycloheximide after a 9-min continuous reactivation (Supplementary Figure S2D). During reactivation, there was no difference between groups (repeated-measures ANOVA; $\mathrm{F}_{(1,12)}=$ $0.805, P=0.39$; Supplementary Figure $\mathrm{S} 2 \mathrm{E}$ ) and within groups $\left(\mathrm{F}_{(2,24)}=1.864, P=0.177\right.$; Supplementary Figure $\mathrm{S} 2 \mathrm{E})$. In the test, rats treated with cycloheximide expressed significantly lower freezing than controls (Student's $t$-test, $t_{(12)}=2.36, P=0.036$; Supplementary Figure S2F), indicating that reconsolidation was disrupted. Also, we infused cycloheximide in a group of rats that underwent extinction (ie, 30-min context re-exposure; Supplementary Figure S2D). During extinction training, both groups were indistinguishable (repeated-measures ANOVA; $\mathrm{F}_{(1,12)}=$ $0.059, P=0.811$; Supplementary Figure S2G) and promoted fear extinction within session $\left(\mathrm{F}_{(5,60)}=13.757, P<0.001\right.$; Supplementary Figure S2G). In the test, cycloheximidetreated rats showed higher freezing levels (Student's $t$-test, $t_{(12)}=3.226, P=0.007$; Supplementary Figure S2H), indicating that extinction consolidation was disrupted. Hence, both 3- and 9-min reactivation induces the protein synthesisdependent reconsolidation process, whereas it requires a 30-min context re-exposure to induce extinction.

Next, to directly investigate whether memory emotional updating described in the experiments 1-3 were mediated by reconsolidation, we injected subcutaneously the LVGCC antagonist nimodipine or its vehicle $30 \mathrm{~min}$ before each reactivation session (Figure 6a).

During reactivations (Figure 6b), repeated-measures ANOVA revealed a significant difference among groups $\left(\mathrm{F}_{(1,35)}=78.34, P<0.001\right)$, demonstrating that the appetitive stimulus was able to hinder freezing responses. There was

Figure 6 Memory valence updating involves reconsolidation as it is mediated by LVGCCs and hippocampal by GluN2B-containing NMDA receptors. (a) Schematic representation of the experimental procedures. Fear-conditioned rats were submitted to memory reactivation sessions on days 3, 5, and 7 after training. Reactivations took place in the conditioned context without any food or in the presence of appetitive stimulus. On day I0, all groups were tested for $4 \mathrm{~min}$. (b) Freezing levels during reactivation sessions. Nimodipine or its vehicle was administered systemically 30 min before each reactivation ( $n=10$ per group); (c) Freezing levels during test. (d) Freezing levels during reactivation sessions. Ifenprodil (appetitive stimulus group, $n=6$; control group, $n=7$ ) or its vehicle (appetitive stimulus group, $n=5$; control group, $n=6$ ) was infused into the CAl region of hippocampus 15 min before each reactivation session. (e) Freezing levels during test. ${ }^{a}$ Significantly different from the control group $(P<0.05$, ANOVA followed by Tukey's post-hoc test). no effect of drug $\left(\mathrm{F}_{(1,35)}=0,26, P=0.61\right)$ or group $\times$ drug interaction $\left(F_{1,35}=0,02, P=0.88\right)$. Thus nimodipine does not affect memory retrieval per se.
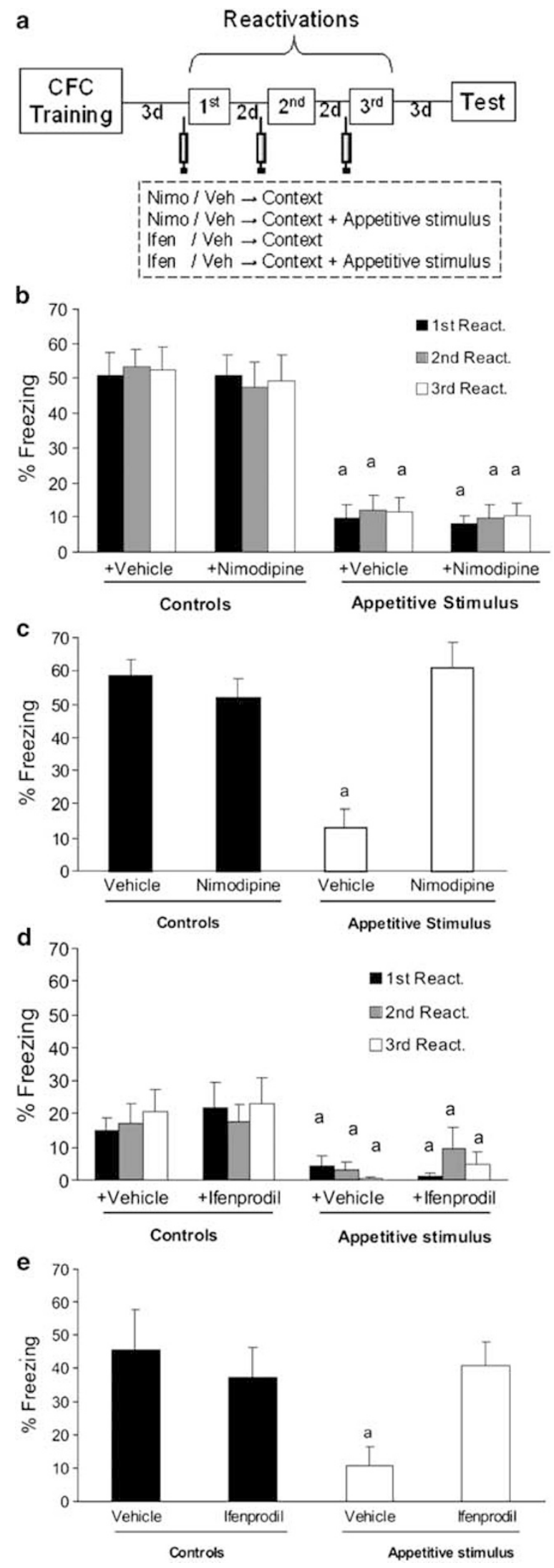
In the test (Figure 6c), two-way ANOVA showed significant effects of group, drug, and group $\times$ drug interaction (group: $\mathrm{F}_{(1,35)}=9.24, \quad P=0.004 ; \quad$ drug: $\mathrm{F}_{(1,35)}=11.8$, $P=0.002$, group $\times$ drug: $\mathrm{F}_{(1,35)}=20.66, P<0.001$.) Tukey's post-hoc test indicated that subjects from the appetitive stimulus group treated with vehicle differed from all the other groups $(P<0.001)$. Accordingly, blocking the L-VGCC during reactivation prevented fear to be attenuated, showing that the memory content emotional updating relies on the LVGCC-dependent destabilization/reconsolidation process.

As memory ages, it becomes resistant to reconsolidation (Suzuki et al, 2004). Hence, we evaluated whether a remote memory could be updated with concomitant appetitive information. First, animals were trained and reactivated 30 days after training Cycloheximide was injected immediately after the reactivation session (Supplementary Figure S3A). During reactivation, there was no difference between groups (Student's $t$-test, $t_{(11)}=0.452, \quad P=0.66$; Supplementary Figure S3B). In the test, cycloheximide-treated group did not show any difference from the controls (Student's $t$-test, $t_{(11)}=0.059, P=0.954$; Supplementary Figure S3C), indicating that the remote memory have not reconsolidated. Next, fear-conditioned animals were submitted on days 30 , 32 , and 34 after training to reactivation sessions in the presence, or not, of the appetitive stimulus (Supplementary Figure S3D). During reactivations, repeated-measures ANOVA showed that the appetitive stimulus group exhibited lower freezing levels than controls $\left(\mathrm{F}_{(1,13)}=13.24, P=0.003\right.$; Supplementary Figure S3E). In the test, however, fear expression of the appetitive stimulus group was not different from the control levels (Student's $t$-test, $t_{(13)}=0.458, P=0.654$; Supplementary Figure S3F). Accordingly, a remote memory should be resistant to reconsolidation and, as a consequence, should also not be amenable to updating.

The hippocampus is a central brain structure with respect to contextual fear memory reconsolidation (Winocur et al, 2009), and the $\mathrm{NMDA}_{\mathrm{R}}$ containing GluN2B subunit is essential for the reactivation-induced memory transition from a stable to a labile state (Ben Mamou et al, 2006; Milton et al, 2013). In order to test this aspect, we infused the selective GluN2B antagonist ifenprodil bilaterally into the CA1 hippocampal region $15 \mathrm{~min}$ before each reactivation.

Compared with all the other experimental groups, the observed effect follows the same general pattern verified in Figure $6 \mathrm{~b}$ and c. During reactivations (Figure 6d), repeatedmeasures ANOVA revealed a significant effect of groups $\left(\mathrm{F}_{(1,19)}=12.49, P=0.002\right)$ but no effect of drug $\left(\mathrm{F}_{(1,19)}=\right.$ $0.45, P=0.51)$ or group $\times$ drug interaction $\left(\mathrm{F}_{(1,19)}=0.007\right.$, $P=0.93$ ). In the test session (Figure 6e), two-way ANOVA revealed marginal group $\times$ drug interaction $\left(\mathrm{F}_{(1,19)}=4.39\right.$, $P=0.050)$. Tukey's post-hoc showed that animals from the appetitive stimulus group receiving vehicle expressed less freezing than those that received ifenprodil $(P=0.035)$ and controls $(P=0.016)$. The lower freezing levels displayed in Figure $6 \mathrm{~d}$ experiment seems to be just a local effect-probably due to the immobilization procedure needed for the drug infusion-as in the test session, where no previous immobilization was necessary, freezing levels remained similar to that observed in the other experiments.
Thus the hippocampal Glun2B antagonism did not affect fear expression during reactivations, but prevented memory to be updated. This result shows that NMDA-related hippocampal plasticity has a critical role in linking hedonic information to an already consolidated contextual fear memory trace.

\section{DISCUSSION}

In the present study, we evaluate the emotional updating of aversive memories through the concomitant presence of hedonic information during reconsolidation. Our results demonstrate that providing stimulus of positive emotional valence during multiple reactivations allows fear memory to be updated to a less aversive level. This procedure resulted in a decreased fear expression when tested in the context previously associated with the footshocks. Moreover, it prevented reinstatement (Experiment 1), spontaneous recovery (Experiment 2), and rapid reaquisition (Experiment 3). Reactivation in the original context was required for the observed effect (Experiment 4), and the concomitant appetitive stimuli relied on its hedonic content to induce updating, as the effect seemed to be dissociated with its nutritional/energetic properties (Experiment 5). We have also found that incorporation of hedonic information to the memory trace is dependent on the destabilizationreconsolidation process, as the phenomenon was prevented both by i.p. injection of the LVGCC antagonist nimodipine and the intrahippocampal infusion of the GluN2B antagonist and is constrained by the memory age boundary condition (Experiment 6).

Taste and smell of food are universal stimuli that produce a hedonic experience, one of the multiple concurrent components of reward (Berridge and Kringelbach, 2013). Previous works have shown that reconsolidation allows memory content to be updated through the incorporation of new information into preexisting memories (for reviews, see Lee, 2009; Besnard, 2012). Also, it has been shown that that an appetitive memory can be updated with aversive information, indicating that memory valence can be changed through reconsolidation (Olshavsky et al, 2013b). Here we have demonstrated for the first time that fear memory can be updated with concomitant appetitive information, retuning its emotional valence. Interestingly, two recent papers have shown that animals with distinct orienting phenotypes respond differently to appetitive and aversive memory updating (Olshavsky et al, 2013a, b). Here the updating process happened only when chocolate or cereal were presented but not with sugar or a non-edible neutral stimulus. We hypothesize that chocolate and cereal have a more salient hedonic value than the others, allowing for memory updating to a less aversive level.

During conditioning, the acquired context-shock association led to the consolidation of an emotional memory with negative valence. However, reactivating memory concomitantly with an appetitive stimulus resulted in a contextappetitive stimulus association that changed memory emotional valence to a less aversive state. Indeed, when the appetitive stimulus was presented in a different context, such association did not occur (Experiment 4). 
Noteworthy, during reactivations the freezing behavior was decreased when appetitive stimulus was present, and somehow this lower emotional state of fear was incorporated into the memory trace. Thus animals kept expressing low freezing levels, even in a posterior test (conducted without appetitive stimuli). One could argue that such effects might be attributed to the nutritional/energetic content of the ingested foods. However, glucose injection before reactivation or chocolate intake in a non-contingent place immediately after reactivation showed no effect on fear expression in the test (Experiment 5). These results show that the fear attenuation effect is not related to the ingested food compounds. However, the lack of effect of glucose here does not rule out the possibility that this substance may affect memory reconsolidation in a different experimental design.

Interestingly, memory was updated to a less aversive level either when the appetitive stimulus was presented during three reactivation sessions of 3 min (Figures 1-3) or during a single 9-min reactivation session (Supplementary Figure S1D-J) However, a single 3-min reactivation was ineffective in hindering fear (Supplementary Figure S1A-C). It is an apparent contradiction as both 3- and 9-min reactivation sessions were able to induce reconsolidation, as indicated by the disruptive effect of the postreactivation infusion of the protein synthesis inhibitor cycloheximide (Supplementary Figure S2A-F). However, it is important to notice that an intervention such as the protein synthesis inhibitor infusion have a dramatic disruptive effect upon memory. On the other hand, the incorporation of appetitive information appears to have a more subtle impact in comparison to the cycloheximide-induced effect. Hence, we hypothesized that the single 3 -min reactivation session induces the incorporation of some degree of appetitive information, but this 'partial' updating of memory content was not sufficient to retune the overall valence of memory to result in an observable change in behavior. Accordingly, multiple 3-min reactivations or a prolonged 9-min reactivation were required to induce a profound re-evaluation of memory content.

The most common therapy in treating fear-related disorders comes from cognitive behavioral therapies that typically involve extinction-based approaches (Rothbaum and Davis, 2003). However, the fear reduction achieved with extinction and other retroactive interference paradigms such as counterconditioning are not permanent as these procedures do not erase nor modify the original fear memory (Bouton, 2002). Rather, they lead to the formation of a new inhibitory memory that suppress the expression of the initial trace (Bouton et al, 2012). On the other hand, modifications on memory content through reconsolidation may allow for permanent changes in fear expression. This idea was reported to be possible in previous studies showing persistent fear suppression by providing an extinction session during the reconsolidation window (Clem and Huganir, 2010; Monfils et al, 2009; Schiller et al, 2010). It has been shown that the mechanism behind the reactivation-extinction updating involves the mediation of hippocampal GluA2-containing AMPA receptor endocitosis (Rao-Ruiz et al, 2011). In this study, we developed a different updating approach by adding information with positive valence during memory reactivation. In order to evaluate whether our updating protocol induces updating of memory content, or some sort of new learning is taking place, we investigated whether the aversive context-shock association could re-emerge. We found that the reactivation + appetitive stimulus procedure resulted in resilient fear attenuation that was not amenable to reinstatement (Experiment 1), spontaneous recovery (Experiment 2), and rapid reacquisition (Experiment 3), therefore indicating a permanent modification of the memory content.

If a reactivated memory becomes labile and requires reconsolidation in order to persist, then blocking destabilization should also prevent reconsolidation. Previous works showed that memory destabilization/reactivation, but not consolidation or retrieval, depends on the LVGCC and on the GluN2B-containing NMDA receptors. In this work, we have shown that nimodipine (Suzuki et al, 2008; Flavell et al, 2011; De Oliveira Alvares et al, 2013) and ifenprodil (Ben Mamou et al, 2006; Milton et al, 2013) were both able to block memory destabilization and prevent reconsolidation. We found that both systemic nimodipine and intrahippocampal ifenprodil infused before the reactivation sessions were able to prevent the fear attenuation, indicating that such effect is indeed mediated by reconsolidation (Experiment 6).

Memory become increasingly resistant to reconsolidation with age (Bustos et al, 2009; Frankland et al, 2006; Milekic and Alberini, 2002; Suzuki et al, 2004). Likewise, here we found that protein synthesis inhibition after reactivation disrupts recent (Supplementary Figure S2A-F) but not remote memory (Supplemenatry Figure S3A-C). We speculated that our reconsolidation-based protocol would also be constrained by this boundary condition. Indeed, our updating protocol failed to promote fear attenuation of 30days-old memory (Supplemenatry Figure S3D-F). This could present an important limitation for the treatment of old traumata. However, the fact that older memories are more resistant to be destabilized does not imply that they cannot undergo reconsolidation (Bustos et al, 2009; Suzuki et al, 2004; Winters et al, 2009). For instance, Gräff et al (2014) showed that the reactivation-extinction procedure fails to attenuate remote fear memories. Remarkably, they found that remote memories can be updated by pharmacologically upregulating neuroplasticityrelated genes. In addition, older memories can undergo reconsolidation through longer reactivation sessions (Bustos et al, 2009; Suzuki et al, 2004). Hence, memory age is an important boundary condition to reconsolidation-mediated updating, but it can be circumvented by pharmacological manipulations and possibly by other methodological adaptations (such as more intense reactivation sessions).

Several studies have shown that fear memories can be weakened by blocking reconsolidation with different drugs (Nader et al, 2000; Debiec and LeDoux, 2006; Lee et al, 2006; Besnard et al, 2012). However, these pharmacological tools usually cannot be readily administered to humans. In agreement with previous reports (Flavell et al, 2011; Monfils et al, 2009; Olshavsky et al, 2013a; Schiller et al, 2010), our results suggest that fear memory can be permanently attenuated without employing drugs or more invasive methodologies. In summary, our findings demonstrate that fear memory may be somehow 'reinterpreted' by the 
presence of an appetitive stimulus during reactivation. This procedure seems to promote a re-evaluation of the contextual fear memory, turning it into a less threatening experience. The ability to shape emotional content of memories through reconsolidation represent a promising preclinical avenue, leading to putative treatments of fear and anxiety disorders such as PTSD or phobias. Our findings also suggest that the development of new nonpharmacological therapeutic approaches may be quite effective in treating fear-related disorders.

\section{FUNDING AND DISCLOSURE}

This work was supported by fellowships and grants from CAPES (MEC), CNPq (MCT), PROPESQ (UFRGS), and FINEP ("Rede Instituto Brasileiro de Neurociências," IBNNet, No. 01.06.0842-00). All the authors declare having no other source of compensation besides primary institution and federal funding. They also report no biomedical financial interests or potential conflicts of interest relevant to the subject matter of this article.

\section{ACKNOWLEDGEMENTS}

We acknowledge Mrs Zelma Regina $\mathrm{V}$ de Almeida for her kind technical assistance and Dr Carla Dalmaz and Dr Maria Elisa Calcagnotto (Depto Biochemisty, ICBS) for their relevant contributions to this paper.

\section{REFERENCES}

Archbold GEB, Bouton ME, Nader K (2010). Evidence for the persistence of contextual fear memories following immediate extinction. Eur J Neurosci 31: 1303-1311.

Berridge KC, Kringelbach ML (2013). Neuroscience of affect: brain mechanisms of pleasure and displeasure. Curr Opin Neurobiol 23: 294-303.

Besnard A (2012). A model of hippocampal competition between new learning and memory updating. J Neurosci 32: 3281-3283.

Besnard A, Caboche J, Laroche S (2012). Reconsolidation of memory: a decade of debate. Prog Neurobiol 99: 61-80.

Blundell J, Kouser M, Powell CM (2008). Systemic inhibition of mammalian target of rapamycin inhibits fear memory reconsolidation. Neurobiol Learn Mem 90: 28-35.

Bouton ME (2002). Context, ambiguity, and unlearning: sources of relapse after behavioral extinction four mechanisms of relapse. Biol Pschiatry 52: 976-986.

Bouton ME, Westbrook RF, Corcoran Ka, Maren S (2006). Contextual and temporal modulation of extinction: behavioral and biological mechanisms. Biol Psychiatry 60: 352-360.

Bouton ME, Winterbauer NE, Todd TP (2012). Relapse processes after the extinction of instrumental learning: renewal, resurgence, and reacquisition. Behav Processes 90: 130-141.

Bustos SG, Maldonado H, Molina Va (2009). Disruptive effect of midazolam on fear memory reconsolidation: decisive influence of reactivation time span and memory age. Neuropsychopharmacology 34: 446-457.

Clem RL, Huganir RL (2010). Calcium-permeable AMPA receptor dynamics mediate fear memory erasure. Science 330: 1108-1112.

Cukor J, Spitalnick J, Difede J, Rizzo A, Rothbaum BO (2009). Emerging treatments for PTSD. Clin Psychol Rev 29: 715-726.

De Oliveira Alvares L, Crestani aP, Cassini LF, Haubrich J, Santana F, Quillfeldt Ja (2013). Reactivation enables memory updating, precision-keeping and strengthening: exploring the possible biological roles of reconsolidation. Neuroscience 244: 42-48.

De Oliveira Alvares L, Einarsson EÖ, Santana F, Crestani AP, Haubrich J, Cassini LF et al (2012). Periodically reactivated context memory retains its precision and dependence on the hippocampus. Hippocampus 22: 1092-1095.

Debiec J, LeDoux JE (2006). Noradrenergic signaling in the amygdala contributes to the reconsolidation of fear memory: treatment implications for PTSD. Ann NY Acad Sci 1071: 521-524.

Díaz-Mataix L, Debiec J, LeDoux JE, Doyère V (2011). Once the US-triggered reconsolidation of fear memory and its CS neural representation is disrupted, a rein- statement procedure does not lead to their recovery. J Neurosci 31: 9538-9543.

Doyère V, Debiec J, Monfils M-H, Schafe GE, LeDoux JE (2007). Synapse-specific reconsolidation of distinct fear memories in the lateral amygdala. Nat Neurosci 10: 414-416.

Flavell CR, Barber DJ, Lee JLC (2011). Behavioural memory reconsolidation of food and fear memories. Nat Commun 2: 504 .

Forcato C, Rodríguez MLC, Pedreira ME, Maldonado H (2010). Reconsolidation in humans opens up declarative memory to the entrance of new information. Neurobiol Learn Mem 93: 77-84.

Frankland PW, Ding H-K, Takahashi E, Suzuki A, Kida S, Silva AJ (2006). Stability of recent and remote contextual fear memory. Learn Mem 13: 451-457.

Gamache K, Pitman RK, Nader K (2012). Preclinical evaluation of reconsolidation blockade by clonidine as a potential novel treatment for posttraumatic stress disorder. Neuropsychopharmacology 37: 2789-2796.

Gold PE (1986). Glucose modulation of memory storage processing. Behav Neural Biol 45: 342-349.

Gold PE, Korol DL (2012). Making memories matter. Front Integr Neurosci 6: 116.

Gräff J, Joseph NF, Horn ME, Samiei A, Meng J, Seo J et al (2014). Epigenetic priming of memory updating during reconsolidation to attenuate remote fear memories. Cell 156: 261-276.

Gross CT, Canteras NS (2012). The many paths to fear. Nat Rev Neurosci 13: 651-658.

Hupbach A, Gomez R, Hardt O, Nadel L (2007). Reconsolidation of episodic memories: a subtle reminder triggers integration of new information. Learn Mem 14: 47-53.

Lee JLC (2009). Reconsolidation: maintaining memory relevance. Trends Neurosci 32: 413-420.

Lee JLC, Milton AL, Everitt BJ (2006). Reconsolidation and extinction of conditioned fear: inhibition and potentiation. J Neurosci 26: 10051-10056.

Mamou C Ben, Gamache K, Nader K (2006). NMDA receptors are critical for unleashing consolidated auditory fear memories. Nat Neurosci 9: 1237-1239.

Messier C (2004). Glucose improvement of memory: a review. Eur J Pharmacol 490: 33-57.

Milekic MH, Alberini CM (2002). Temporally graded requirement for protein synthesis following memory reactivation. Neuron 36: 521-525.

Milton AL, Merlo E, Ratano P, Gregory BL, Dumbreck JK, Everitt BJ (2013). Double dissociation of the requirement for GluN2B- and GluN2A-containing NMDA receptors in the destabilization and restabilization of a reconsolidating memory. J Neurosci 33: 1109-1115.

Monfils M-H, Cowansage KK, Klann E, LeDoux JE (2009). Extinction-reconsolidation boundaries: key to persistent attenuation of fear memories. Science 324: 951-955.

Nader K, Schafe GE, Le Doux JE (2000). Fear memories require protein synthesis in the amygdala for reconsolidation after retrieval. Nature 406: 722-726.

Olshavsky ME, Jones CE, Lee HJ, Monfils M-H (2013a). Appetitive behavioral traits and stimulus intensity influence maintenance of conditioned fear. Front Behav Neurosci 7: 179. 
Olshavsky ME, Song BJ, Powell DJ, Jones CE, Monfils M-H, Lee HJ (2013b). Updating appetitive memory during reconsolidation window: critical role of cue-directed behavior and amygdala central nucleus. Front Behav Neurosci 7: 186.

Parsons RG, Ressler KJ (2013). Implications of memory modulation for post-traumatic stress and fear disorders. Nat Neurosci 16: 146-153.

Rao-Ruiz P, Rotaru DC, Van Der Loo RJ, Mansvelder HD, Stiedl O, Smit $\mathrm{AB}$ et al (2011). Retrieval-specific endocytosis of GluA2AMPARs underlies adaptive reconsolidation of contextual fear. Nat Neurosci 14: 1302-1308.

Rescorla Ra, Heth CD (1975). Reinstatement of fear to an extinguished conditioned stimulus. J Exp Psychol Anim Behav Process 1: 88-96.

Robinson MJF, Berridge KC (2013). Instant transformation of learned repulsion into motivational 'wanting'. Curr Biol 23: 282-289.

Rodriguez-Ortiz CJ, la Cruz V De, Gutiérrez R, Bermudez-Rattoni F (2005). Protein synthesis underlies post-retrieval memory consolidation to a restricted degree only when updated information is obtained. Learn Mem 12: 533-537.

Rothbaum BO, Davis M (2003). Applying learning principles to the treatment of post-trauma reactions. Ann NY Acad Sci 1008: $112-121$.

Schiller D, Monfils M-H, Raio CM, Johnson DC, Ledoux JE, Phelps Ea (2010). Preventing the return of fear in humans using reconsolidation update mechanisms. Nature 463: 49-53.
Sierra RO, Cassini LF, Santana F, Crestani AP, Duran JM, Haubrich J et al (2013). Reconsolidation may incorporate statedependency into previously consolidated memories. Learn Mem 20: 379-387.

Steckler T, Risbrough V (2012). Pharmacological treatment of PTSD_established and new approaches. Neuropharmacology 62: 617-627.

Stern CAJ, Gazarini L, Takahashi RN, Guimarães FS, Bertoglio LJ (2012). On disruption of fear memory by reconsolidation blockade: evidence from cannabidiol treatment. Neuropsychopharmacology 37: 1-11.

Suzuki A, Josselyn Sa, Frankland PW, Masushige S, Silva AJ, Kida S (2004). Memory reconsolidation and extinction have distinct temporal and biochemical signatures. J Neurosci 24: 4787-4795.

Suzuki A, Mukawa T, Tsukagoshi A, Frankland PW, Kida S (2008). Activation of LVGCCs and CB1 receptors required for destabilization of reactivated contextual fear memories. Learn Mem 15: 426-433.

Winocur G, Frankland PW, Sekeres M, Fogel S, Moscovitch M (2009). Changes in context-specificity during memory reconsolidation: selective effects of hippocampal lesions. Learn Mem 16: 722-729.

Winters BD, Tucci MC, DaCosta-Furtado M (2009). Older and stronger object memories are selectively destabilized by reactivation in the presence of new information. Learn Mem 16: 545-553.

Supplementary Information accompanies the paper on the Neuropsychopharmacology website (http://www.nature.com/npp) 